\title{
Random dopant model for three-dimensional drift-diffusion simulations in metal-oxide-semiconductor field-effect-transistors
}

\author{
Nobuyuki Sano a) and Masaaki Tomizawa ${ }^{\text {b) }}$ \\ Institute of Applied Physics, University of Tsukuba, Tsukuba, Ibaraki 305-8573, Japan
}

(Received 9 April 2001; accepted for publication 25 July 2001)

\begin{abstract}
We investigate the dopant model employed in drift-diffusion device simulations for the study of statistical threshold voltage variations associated with discrete random dopants. It is pointed out that the conventional dopant model, when extended to the extreme "atomistic" regime, becomes physically inconsistent with the length-scale presumed in drift-diffusion simulations. Splitting the Coulomb potential of localized dopants between the long-range and short-range parts, we propose a dopant model appropriate for three-dimensional drift-diffusion simulations. (C) 2001 American Institute of Physics. [DOI: 10.1063/1.1406980]
\end{abstract}

The problem associated with threshold voltage $\left(V_{\mathrm{th}}\right)$ variations caused by discrete random dopants was pointed out more than a few decades ago ${ }^{1}$ and is now becoming a real problem in ultrasmall metal oxide semiconductor field effect transistors (MOSFETs). ${ }^{2}$ The problem has been numerically studied with the conventional two-dimensional (2D) and/or three-dimensional (3D) drift-diffusion (DD) simulations following the pioneering works of Nishinohara et $a .^{3}$ and Wong and Taur, ${ }^{4}$ in which the dopant density at each mesh node varies in accordance with the number of dopants included in the mesh. This approach has been extended to the extreme "atomistic" regime, where most meshes contain no dopant or, at most, one dopant. ${ }^{5-8}$ Indeed, this is the situation actually taken place in real sub-100 nm MOSFETs.

However, it is not certain whether such an extension of the conventional dopant model to the atomistic regime is consistent with the physics presumed in the DD simulation scheme. In fact, according to our recent study, ${ }^{9,10}$ threshold characteristics in sub-100 $\mathrm{nm}$ MOSFETs could be drastically changed when all dopants inside the entire device regions are treated as being discrete and, thus, atomistic. It is, therefore, the purpose of the present letter to clarify this controversial issue from the viewpoint of device physics. It is pointed out that, when the conventional dopant model is extended to the extreme atomistic regime, the electrostatic Coulomb potential becomes physically inconsistent in its length scale with the potential presumed in DD device simulations. The split of the Coulomb potential of discretized dopants between the long-range and short-range parts is critical for quantitative investigations of statistical $V_{\text {th }}$ variations of ultrasmall MOSFETs.

It is important to note that uniform substrate dopant actually implies uniform dopant arrangements on a macroscopic scale, whose length scale is usually equivalent to the mesh spacing employed in device simulations. On the other hand, the random dopant variations leading to $V_{\text {th }}$ fluctuations are associated with microscopic nonuniformity of dopant arrangements. Hence, the key point to study dopant fluc-

\footnotetext{
${ }^{a)}$ Electronic mail: sano@esys.tsukuba.ac.jp

b) NTT Lifestyle and Environmental Laboratories, 3-1 MorinosatoWakamiya, Atsugi, Kanagawa 243-0198, Japan.
}

tuations in ultrasmall devices lies in the question how such microscopic nonuniformity of the dopant arrangement inside the device could be introduced in conventional DD simulations.

In order to take into account such nonuniformity, the following scheme is usually employed in DD simulations: The average number of dopants included in each mesh is estimated from the macroscopic dopant profile already known from experiments and/or process simulations. The number of dopants at each mesh node is then determined from the Poisson distribution with the average number of dopants found above. With this method, one can easily introduce the dopant density variations in conventional DD simulations. In many cases where device size is relatively large and/or of 2D DD simulations, the number of dopants included in each mesh region exceeds unity. Therefore, the dopant density at each mesh node is given by a finite number and does not change abruptly. In other words, dopants may be regarded as continuous jellium and, thus, the potential correctly represents the smoothly changing band edge. On the other hand, when this dopant model is extended to the extreme atomistic case, the dopant density at each mesh node changes abruptly and behaves like the $\delta$-function. As a result, the electrostatic potential is the Coulomb potential and singular at the point of each localized dopant. This is interpreted as follows. In large devices, the mesh spacing is usually greater than the mean separation of dopants and, therefore, the short-range part of the Coulomb potential of each localized dopant is implicitly eliminated. ${ }^{11}$ On the other hand, the mesh spacing is always smaller than the mean separation of dopants in the atomistic devices, the short-range part of the Coulomb potential, in addition to the long-range part, is explicitly included. Consequently, the electric potential obtained from the atomistic dopant becomes a full potential rather than a gradually changing long-range potential.

The question is which potential, the full potential or long-range potential, is appropriate for classical DD device simulations. When the full potential, as given by the atomistic dopants, is employed in DD simulations, the majority carriers near the localized dopants are strongly trapped by the Coulomb potential and the potential of each dopant is always screened regardless of applied gate voltages. This is 
equivalent to saying that the charge of each dopant is effectively reduced. Since the potential profile of the dopant is dependent on the mesh employed, the amount of the screening charge around the dopant is also dependent on the mesh; The smaller the mesh is, the stronger the screening. This may explain why the negative $V_{\text {th }}$ shift is observed in atomistic DD simulations ${ }^{5-8}$ because of the reduction of the dopant density in the substrate. This is, however, quantum mechanically inconsistent. Quantum mechanics shows that the shallow bound states are usually formed very close to the band edge in $\mathrm{Si}$ and, at room temperature, most carriers are relieved from such bound states. ${ }^{12}$ Thus, the dopants are barely screened under the depletion condition. In addition, according to the modified Hartree approximation, ${ }^{13}$ the majority carrier density under spatially localized dopants is rather uniform in equilibrium when the dopant density is large. Otherwise, the number of majority carriers exceeds that of ionized dopants and the charge neutrality condition is violated. Therefore, the amount of the screening charge, or equivalently the band bending, can not be so large under the flatband condition as in the atomistic simulations. Hence, we may conclude that to split the Coulomb potential of ionized dopants between the long-range and short-range parts is essential, and it is the long-range part that should be incorporated in classical DD simulators.

In order to split the Coulomb potential of ionized dopants between the long-range and short-range parts, we have looked at the number density of each localized dopant. The number density of each dopant, rather than the electric potential, is explicitly separated between the long-range and short-range parts. The long-range part of the number density $\rho_{\mathrm{ac}}^{\text {long }}(r)$ for a single acceptor located at the origin $(\mathbf{r}=0)$ is then given by ${ }^{14}$

$$
\rho_{\mathrm{ac}}^{\text {long }}(r)=\frac{k_{c}^{3}}{2 \pi^{2}} \frac{\sin \left(k_{c} r\right)-\left(k_{c} r\right) \cos \left(k_{c} r\right)}{\left(k_{c} r\right)^{3}},
$$

where $k_{c}$ is the cut-off parameter by which the split of the long-range and short-range parts of the number density is made. We would like to stress that Eq. (1) is identical to the expression obtained from the long-range part of the Coulomb potential. ${ }^{15}$ Physically, $k_{c}$ is related with the screening length of the Conwell-Weisskopf model ${ }^{16}$ and should be given by a half of the mean separation of dopants. In the present DD simulations, however, it is treated as a fitting parameter. Figure 1 shows the long-range and short-range parts of the number density of a single acceptor. Notice that the singularity at the origin is properly removed for the long-range part. As a consequence, $\rho_{\text {ac }}^{\text {long }}(r)$ spreads over the length $1 / k_{c}$. This is a sharp contrast to the case of atomistic dopants which shows the $\delta$-function behavior.

The validity of the present dopant model is checked by simulating device characteristics of relatively large MOSFETs: When the device size is large so that the substrate dopant distribution is regarded as macroscopically uniform because of averaging over the device dimensions, the present dopant model must reproduce the same device characteristics as those of the conventional 2D DD simulations. Figure 2 shows the threshold characteristics for $n$-channel MOSFETs with the two different device sizes: (a) the gate length $L_{g}=50 \mathrm{~nm}$ and the device width $W=500 \mathrm{~nm}$ and (b)

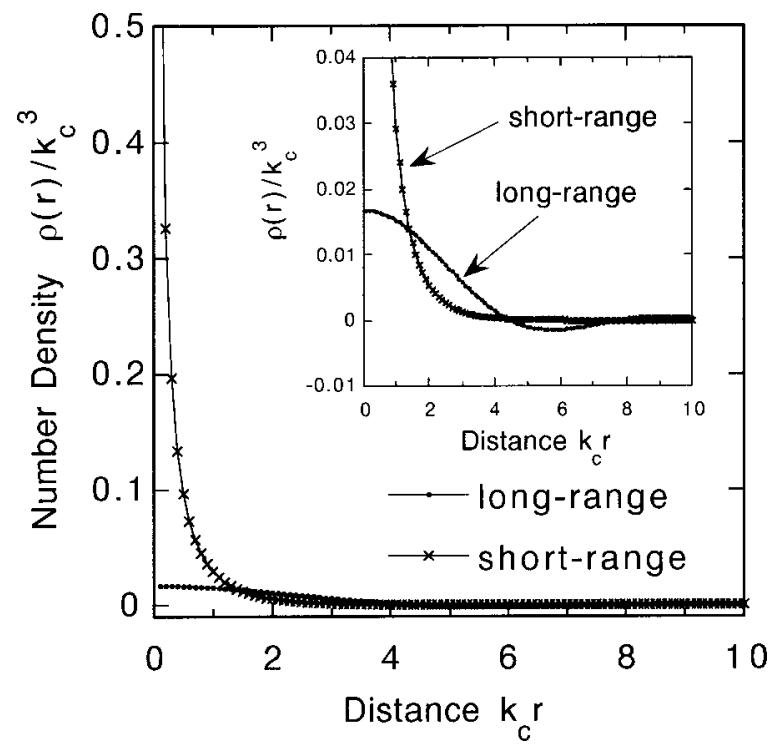

FIG. 1. Long-range and short-range parts of the number density of a single acceptor located at the origin are shown.

$L_{g}=50 \mathrm{~nm}$ and $W=50 \mathrm{~nm}$. The macroscopic dopant density $N_{\text {ac }}$ is assumed to be uniform $\left(N_{\mathrm{ac}}=10^{18} \mathrm{~cm}^{-3}\right)$ so that $1 / k_{c}=N_{\mathrm{ac}}^{-1 / 3} / 2=5 \mathrm{~nm}$. The threshold characteristics are evaluated by the 3D DD simulations with the present dopant model and by the conventional 2D DD simulations. In the case of the large MOSFET, the results obtained from the 3D and 2D simulations coincide very well regardless of the gate voltages $V_{g}$. This agreement is actually surprising because it was unnecessary to adjust the parameter $k_{c}$. This implies

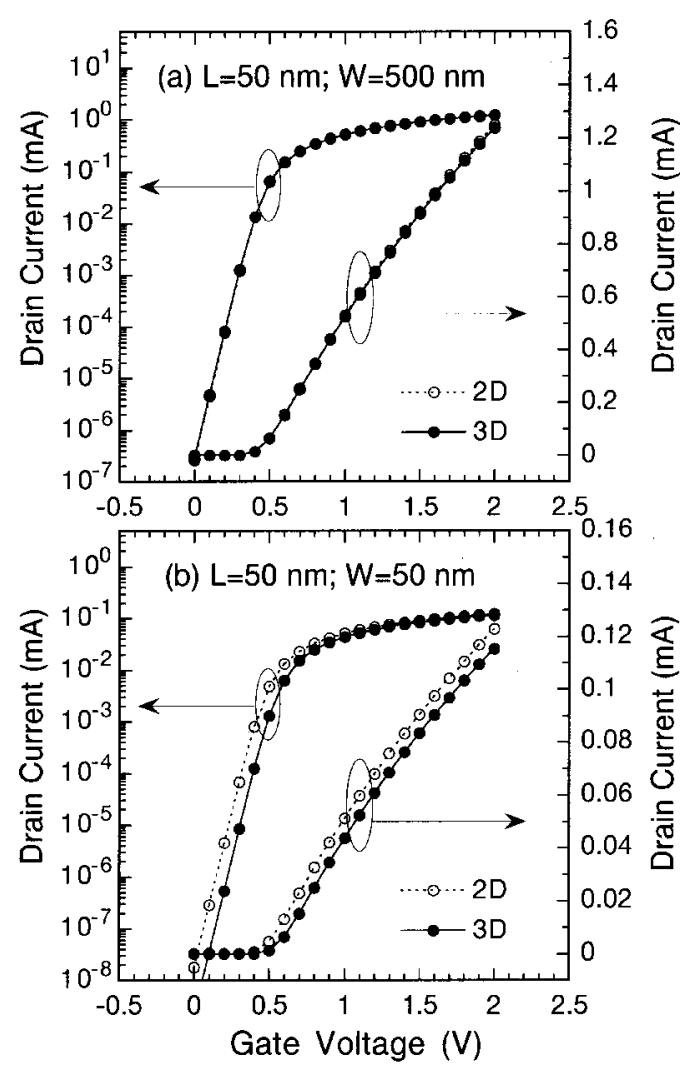

FIG. 2. Threshold characteristics for n-channel MOSFETs with (a) $L_{g}$ $=50 \mathrm{~nm}$ and $W=500 \mathrm{~nm}$ and (b) $L_{g}=50 \mathrm{~nm}$ and $W=50 \mathrm{~nm}$ are shown. The solid (dotted) lines represent the results from the 3D (2D) DD simulations. to AIP license or copyright, see http://apl.aip.org/apl/copyright.jsp 


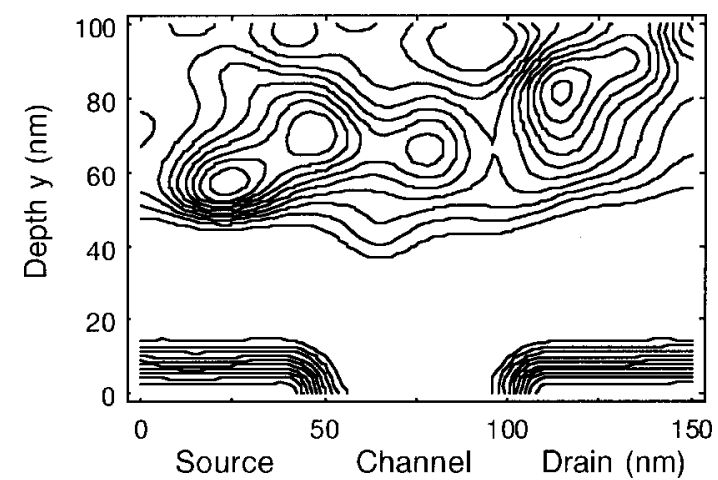

FIG. 3. Contour plots of the hole and electron densities in the plane perpendicular to the gate oxide in the MOSFET with $L_{g}=50 \mathrm{~nm}$ under $V_{g}$ $=0.6 \mathrm{~V}$ are shown.

that with the choice of the present $k_{c}$, the long-range part of the Coulomb potential, that is the amount of band bending, can be properly taken into account. On the other hand, the two results begin to deviate as the device size shrinks and this is a typical example of $V_{\text {th }}$ variations associated with microscopic nonuniformity of discrete dopants. The deviation is much greater in the subthreshold regions and this is consistent with the expectation that the microscopic nonuniformity of dopants would affect transport characteristics more significantly when the carriers penetrate into the deep regions of the substrate. The origin of such threshold variations is well demonstrated in Fig. 3, in which the contour plots of the hole and electron densities in the plane perpendicular to the gate oxide are shown for the MOSFET with $L_{g}=50 \mathrm{~nm}$ under $V_{g}=0.6 \mathrm{~V}$. The edge of the hole density, nearly equivalent to the edge of the depletion region, is greatly deformed due to the microscopically nonuniform arrangement of dopants.

In summary, we investigated the meaning of the discrete dopant model widely used in DD simulators and pointed out that the conventional dopant model, when extended to the extreme atomistic regime, becomes physically inconsistent with the length-scale presumed in DD simulations. A dopant model in which the Coulomb potential is split between the long-range and short-range parts was introduced.

The authors would like to thank C. Hamaguchi, K. Matsuzawa, M. Mukai, N. Nakayama, K. Nishinohara, and S. Odanaka for valuable discussion and encouragement during the study. This work was supported in part by the Semiconductor Technology Academic Research Center (STARC).

${ }^{1}$ B. Honenstein and C. A. Meed, Solid-State Electron. 15, 819 (1972).

${ }^{2}$ T. Tanaka, T. Usuki, T. Futatsugi, Y. Momiyama, and T. Sugii, Tech. Dig. Int. Electron Devices Meet., 271 (2000).

${ }^{3}$ K. Nishinohara, N. Shigyo, and T. Wada, IEEE Trans. Electron Devices 39, 634 (1992).

${ }^{4}$ H. S. Wong and Y. Taur, Tech. Dig. Int. Electron Devices Meet., 705 (1993).

${ }^{5}$ A. Asenov, IEEE Trans. Electron Devices 45, 2505 (1998).

${ }^{6}$ D. Vasileska, W. J. Gross, and D. K. Ferry, in Proceedings of the International Workshop on Computational Electronics, 1998, p. 259.

${ }^{7}$ D. J. Frank, Y. Taur, M. Ieong, and H.-S. P. Wong, in Proceedings of the VLSI Tech. Symposium, 1999, p. 169.

${ }^{8}$ A. Asenov, G. Slavcheva, A. R. Brown, R. Balasubramaniam, and J. H. Davies, Superlattices Microstruct. 27, 215 (2000).

${ }^{9}$ N. Sano and M. Tomizawa, in Ext. Abst. Int. Conf. Solid State Devices and Materials, 2000, p. 216.

${ }^{10}$ N. Sano, K. Matsuzawa, M. Mukai, and N. Nakayama, Tech. Dig. Int. Electron Devices Meet., 275 (2000).

${ }^{11}$ W. J. Gross, D. Vasileska, and D. K. Ferry, IEEE Electron Device Lett. 20, 463 (1999).

${ }^{12}$ J. M. Ziman, Principles of The Theory of Solids (Cambridge University Press, New York, 1972), Sec. 6.4.

${ }^{13}$ C. Kittel, Quantum Theory of Solids (Wiley, New York, 1963), Chap. 5.

${ }^{14}$ This expression is oscillatory at large $r$, but its contribution is negligible if the integral region is sufficiently large. However, the fact that the charge density is broadened is of most importance, rather than its exact expression.

${ }^{15}$ For example, see, E. K. U. Gross, E. Runge, and O. Heinonen, ManyParticle Theory (Hilger, New York, 1991), p. 87.

${ }^{16}$ D. Chattopadhyay and H. J. Queisser, Rev. Mod. Phys. 53, 745 (1981). 